\title{
Correction to: Pharmacists' confidence when providing pharmaceutical care on anticoagulants, a multinational survey
}

\author{
John Papastergiou ${ }^{1,2} \cdot$ Nadir Kheir $^{3} \cdot$ Katerina Ladova $^{4} \cdot$ Silas Rydant $^{5} \cdot$ Fabio De Rango $^{6} \cdot$ Sotiris Antoniou $^{7,8}$. \\ Reka Viola ${ }^{9}$ Maria Dolores Murillo ${ }^{10} \cdot$ Stephane Steurbaut ${ }^{11} \cdot$ Filipa Alves da Costa ${ }^{12,13}$ (1)
}

Published online: 10 January 2018

(c) Springer International Publishing AG, part of Springer Nature 2018

\section{Correction to: Int J Clin Pharm (2017) 39:1282-1290 https://doi.org/10.1007/s11096-017-0551-2}

In the original publication of this article, the article note has been missed and published online. Now the same has been provided in this erratum.

On behalf of the International Pharmacists for Anticoagulation Care Taskforce (iPACT).
The original article can be found online at https://doi.org/10.1007/ s11096-017-0551-2.

Filipa Alves da Costa

filipa.alvesdacosta@ipact.org

1 Leslie Dan Faculty of Pharmacy, University of Toronto, Toronto, ON M4J 1L2, Canada

2 School of Pharmacy, University of Waterloo, 755 Danforth Avenue, Toronto, ON M4J 1L2, Canada

3 College of Pharmacy, Qatar University, PO Box 2713, Doha, Qatar

4 Department of Social and Clinical Pharmacy, Faculty of Pharmacy in Hradec Kralove, Charles University, Akademika Heyrovskeho 1203, 50005 Hradec Kralove, Czech Republic

5 Pharmaceutical Care Division (Meduca), Royal Pharmacists Association of Antwerp (KAVA), Lange Leemstraat 187, 2018 Antwerpen, Belgium

6 Shoppers Drug Mart 1271, 2501 Third Line, Oakville, ON L6M 5A9, Canada
Stephane Steurbaut and Filipa Alves da Costa are joined last authors, and they have contributed equally to this study.

Other iPACT members that have made a contribution to this study are A. Laven, B. van den Bemt, C. Pessanha, D. Griffiths, H. Williams, M.-C. Chaumais, M. Holden, N. O'Hanlon and S. Falamic.
7 Barts Health Centre; Barts Health NHS Trust, London, UK

8 UCL Partners, London, UK

9 Department of Clinical Pharmacy, Faculty of Pharmacy, University of Szeged, Szikrautca 8, Szeged 6724, Hungary

10 Farmacia Fernández Vega C.B., C/Par nº 26 Urbanización Club de Golf. Alcalá de Guadaira, Sevilla 41500, Spain

11 Research Group Clinical Pharmacology \& Clinical Pharmacy (KFAR), Faculty of Medicine and Pharmacy, Vrije Universiteit Brussel, Laarbeeklaan 101, 1090 Brussels, Belgium

12 Centro de Investigação Interdisciplinar Egas Moniz (CiiEM), Campus Universitário, Quinta da Granja, Monte da Caparica, 2829-551 Caparica, Portugal

13 Portuguese Pharmaceutical Society (PPS), Rua da Sociedade Farmacêutica, 18, 1169-075 Lisbon, Portugal 